\title{
Variational Phase Imaging Using the Transport-of-Intensity Equation
}

\author{
Emrah Bostan, Student Member, IEEE, Emmanuel Froustey, Masih Nilchian, Student Member, IEEE, \\ Daniel Sage, and Michael Unser, Fellow, IEEE
}

\begin{abstract}
We introduce a variational phase retrieval algorithm for the imaging of transparent objects. Our formalism is based on the transport-of-intensity equation (TIE), which relates the phase of an optical field to the variation of its intensity along the direction of propagation. TIE practically requires one to record a set of defocus images to measure the variation of intensity. We first investigate the effect of the defocus distance on the retrieved phase map. Based on our analysis, we propose a weighted phase reconstruction algorithm yielding a phase map that minimizes a convex functional. The method is nonlinear and combines different ranges of spatial frequencies-depending on the defocus value of the measurements-in a regularized fashion. The minimization task is solved iteratively via the alternatingdirection method of multipliers. Our simulations outperform commonly used linear and nonlinear TIE solvers. We also illustrate and validate our method on real microscopy data of HeLa cells.
\end{abstract}

Index Terms-Phase retrieval, transport-of-intensity equation, sparse reconstruction, total variation regularization, weightednorm regularization, phase imaging, phase microscope.

\section{INTRODUCTION}

$\mathbf{T}$ HE GENERIC problem of imaging transparent objects is highly relevant to biological research as most cells and thin tissue samples do not absorb light and produce images with very low contrast when observed under a standard bright-field microscope (see Figure 1(a)) [1]. To reveal specific structures in the sample, one can apply staining or use fluorescent dyes and biomarkers. These exogenous contrast agents then allow for the use of advanced light microscopy techniques [2], [3]. These high-end modalities, however, require careful sample preparation and are not well-suited to image live cells (especially over extended periods of time) since the contrast agents can be phototoxic [4]. Also, the observation of the global tissue or cell morphology becomes harder [5]. For these reasons, label-free phase imaging techniques are

Manuscript received June 9, 2015; revised October 21, 2015 and November 30, 2015; accepted December 5, 2015. Date of publication December 17, 2015; date of current version January 7, 2016. The research leading to these results has received funding from the European Research Council under European Union's Seventh Framework Programme (FP7/2007-2013)/ERC grant agreement no 267439. The associate editor coordinating the review of this manuscript and approving it for publication was Prof. Jong Chul Ye. EB and EF contributed equally.

The authors are with the Biomedical Imaging Group, École Polytechnique Fédérale de Lausanne, Lausanne CH-1015, Switzerland (e-mail: emrah.bostan@gmail.com; emmanuel.forustey@epfl.ch; masih.nilchian@epfl.ch; daniel.sage@epfl.ch; michael.unser@epfl.ch).

Color versions of one or more of the figures in this paper are available online at http://ieeexplore.iee.org.

Digital Object Identifier 10.1109/TIP.2015.2509249 needed especially when minimal manipulation of the cell is required (such as in stem cell and drug discovery studies) [6]. While transparent objects have insignificant absorption, they do introduce phase shifts due to variations in the optical path length in the sample. The information about the distribution of the refractive index (hence, about the morphology of the cell) is encoded in the phase. Two conventional imaging modalities that translate the phase shifts into detectable intensity differences are phase contrast microscopy (PC) [7] and differential interference contrast (DIC) microscopy [8]. However, both DIC and PC images are challenging to segment (and to track) due to shade and halo artifacts (see Figure 1). This essentially limits their use since segmentation is a key step in cell biology [9]. Another well-established technique for phase imaging is digital holographic microscopy (DHM) [10], [11]. DHM yields quantitative phase information, and hence shape maps of the object being imaged. This allows for the segmentation of cells and other specific structures. The downside of DHM is that it is a hardware-based solution of higher cost compared to bright-field, DIC, and PC microscopies [12].

In this paper, we consider a low-cost label-free phase imaging approach that uses the transport-of-intensity equation (TIE) [13]. This method has significant advantages: it is a computational method that can be used with either bright-field [14] or DIC [15] microscopes; the resulting phase dispenses with the unwrapping task required by interferometric methods such as DHM [16]. As we shall see later, TIE puts forward a linear mathematical formalism that relates the spatial phase map of the sample to the derivative of its intensity map along the propagation direction. In a practical setup, the phase is recovered by using a set of images acquired at various positions along the optical axis. Hence, TIE simply requires a stack of defocus measurements to obtain the phase information at the infocus plane (i.e., the axial location of the sample).

\section{A. Overview of Previous Approaches}

TIE has been extensively studied in the optics community, where researchers have explored ways to obtain better estimates of the axial derivative of the intensity. The simplest approach has been to capture two images that are slightly above and below the focus plane, followed by a centered finite difference. Various refined models using additional defocused images have also been proposed. In [17], the authors consider computing higher-order terms in the Taylor development of the intensity along the optical axis. The use of finite differences results in a simple linear combination of defocused images, 

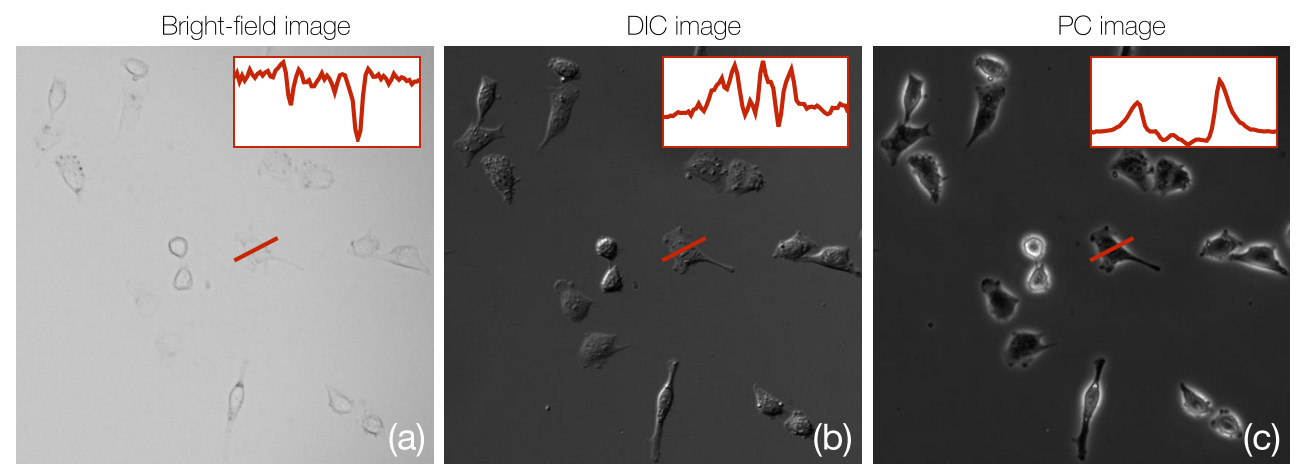

Fig. 1. Imaging the same HeLa cells with different microscopes. Infocus images for the same field of view are obtained by using (a) bright-field, (b) DIC, and (c) PC microscopes. Profiles corresponding to the same line segment are given at the top-right corner of each image.

but the estimate tends to be sensitive to noise. An alternative approach is based on a pixel-wise polynomial fitting of the intensity along the optical axis [17]. Again, it is carried out at the expense of acquiring more images. A variation of this technique is proposed in [18], where wavelet shrinkage is shown to improve the accuracy over the polynomial fitting method. The authors of [19] have presented a framework in which they estimate the axial derivative of the intensity through fitting in the spatial frequency domain. Alternatively, one may combine multiple TIE solutions that are obtained by using different defocus measurements. In [20], two different TIE reconstructions are spectrally weighted by using Gaussian filters. The authors have designed spectral weighting coefficients that minimize the pointwise variance in the frequency domain in [21]. In [22], multiple spectral bands are combined via the Savitzky-Golay differentiation filter. Similarly, bandpass filters that are specific to the defocus distance are used in the frequency domain for the calculation of the phase image [23]. What is common in all of these methods is that they are all linear reconstruction algorithms and that the TIE is solved directly by inverting the model. The latter suggests the use of regularization to stabilize the solutions. Tikhonov [24] and total variation (TV) [25]-[27] regularizations have been considered.

\section{B. Contributions}

Fundamentally, TIE suffers from two problems. First, when the model is inverted, the noise in the measurements is integrated because TIE is a differential form. This typically produces low-frequency errors (such as "cloudy" artifacts) in the reconstructions. Second, the computation of the axial intensity derivative by using centered finite differences necessitates small defocus distances. Deviations from this requirement introduce further errors. As we shall show in the coming sections, these problems are tightly related. Extending our previous research [27], we propose a nonlinear variational method that provides a unifying framework for the resolution of TIE. Our contributions are as follows:

- A joint model that is able to combine the spectral information coming from different defocus distances within a regularized reconstruction framework. The model is built upon identifying reliable frequency ranges as the defocus distance changes.
- The proposal of an iterative algorithm that allows us to efficiently solve the related optimization problem.

- A detailed comparison of our method with common TIE reconstruction algorithms. We show that we achieve better phase reconstructions for both simulated and experimental data. The reconstructed phase images are also validated by comparing them with DHM acquisitions.

\section{Outline}

The paper is organized as follows: In Section II, we explain the underlying physics and introduce two complementary models that constitute the core of our approach. In Section III, we cast the phase reconstruction task as an inverse problem and explain our iterative algorithm. Finally, we provide numerical simulations, real data experiments, and discuss our results in Section IV.

\section{Physical Models}

Consider the problem geometry given in Figure 2. We define $(\boldsymbol{x}, z)$ as our coordinate vector where $\boldsymbol{x}=\left(x_{1}, x_{2}\right) \in \mathbb{R}^{2}$ denotes the spatial location on a transverse plane that is perpendicular to the optical axis $z$. The monochromatic plane wave $\mathrm{e}^{\mathrm{j} k z}$ illuminates a thin object that lies on a bounded domain $\Omega \subset \mathbb{R}^{2}$. The wave function after traversing the object is written as

$$
U(\boldsymbol{x}, z)=U_{A}(\boldsymbol{x}, z) \mathrm{e}^{\mathrm{j} k z},
$$

where $U_{A}$ represents the so-called complex amplitude of $U$, $k=2 \pi / \lambda$ is the wave number with $\lambda$ being the illumination wavelength. The expression of the complex amplitude is given by

$$
U_{A}(\boldsymbol{x}, z)=\sqrt{I(\boldsymbol{x}, z)} \mathrm{e}^{\mathrm{j} \phi(x, z)},
$$

where the real-valued functions $I$ and $\phi$ are the intensity and the phase, respectively.

For convenience, we shall assume that the object is located at the axial position $z=0$. Therefore, the wave field at the object plane is specified by

$$
O(x)=U_{A}(x, 0)=\sqrt{I_{0}(x)} \mathrm{e}^{\mathrm{j} \phi_{0}(x)},
$$

where $\phi_{0}(\boldsymbol{x})=\phi(\boldsymbol{x}, 0)$ corresponds to the spatial phase map of the object. In practice, it is simpler to obtain the intensity of 


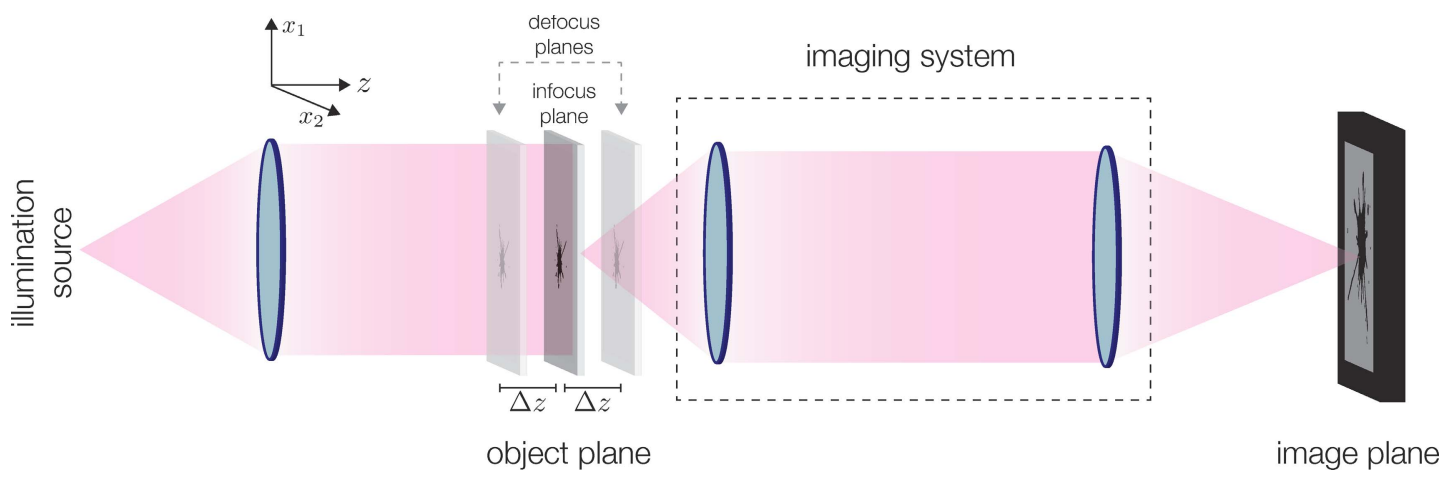

Fig. 2. Representation of the measurement model (and the corresponding problem geometry) in this paper. A transparent object is located at the input plane of an ideal imaging system. A magnified version of the object field is generated at the image plane. Defocus images are captured by moving the object symmetrically around the focus position. The phase map at the infocus plane is then obtained by using the defocus images in (10).

the field. This necessitates establishing a relationship between the phase information, which is lost during acquisition, and the intensity measurements.

\section{A. Transport-of-Intensity Equation}

Suppose the propagation of $U_{A}$ is dominant along the $z$ axis (i.e., the paraxial approximation is valid). Then, the physics of $U_{A}$ is governed by the paraxial wave equation

$$
\left(\nabla_{\perp}^{2}+2 \mathrm{j} k \frac{\partial}{\partial z}\right) U_{A}(\boldsymbol{x}, z)=0,
$$

where $\nabla_{\perp}^{2}$ is the transverse Laplacian operator defined by $\nabla_{\perp}^{2}=\left(\partial^{2} / \partial x_{1}^{2}+\partial^{2} / \partial x_{2}^{2}\right)$.

By multiplying (4) by $U_{A}^{*}$ on the left-hand side, and separating the real and the imaginary parts, Teague [13] has derived two equations. In particular, the imaginary part $^{1}$ specifies the transport-of-intensity equation (TIE)

$$
-k \frac{\partial}{\partial z} I(\boldsymbol{x}, z)=\nabla_{\perp} \bullet\left(I(\boldsymbol{x}, z) \nabla_{\perp} \phi(x, z)\right),
$$

where $\nabla_{\perp}=\left[\begin{array}{lll}\partial / \partial x_{1} & \partial / \partial x_{2}\end{array}\right]^{\mathrm{T}}$ is the transverse gradient operator and $\bullet$ denotes the dot product. We see that (5) is an elliptical second-order partial differential equation that links the phase information to the axial derivative of the intensity of the field. Practically, TIE bears an important outcome: the spatial phase map $\phi$ is computed by measuring the intensity $I$ and its axial derivative $\partial I(\boldsymbol{x}, z) / \partial z$, where the latter can be approximated by finite differences.

We now investigate TIE in relation to our problem settings. We consider an object that is at the input plane of an ideal magnification system, generating a dilated version of the object field at the image plane. As mentioned in Section I, we are interested in imaging unstained biological samples. Such class of objects are modeled as phase-only objects, meaning that they do not significantly absorb or scatter the illuminating field [5]. We further assume that the illumination is uniform

\footnotetext{
${ }^{1}$ The real part provides us with the so-called transport-of-phase equation (TPE). However, TPE requires the measurement of the axial derivative of the phase $\partial \phi / \partial z$. This makes TPE impractical as $\phi$ itself is unknown.
}

so that $I_{0}(\boldsymbol{x})$ is constant: $I_{0}(\boldsymbol{x})=J_{0}$. Therefore, (5) is rewritten as

$$
-\left.\frac{k}{J_{0}} \frac{\partial}{\partial z} I(\boldsymbol{x}, z)\right|_{z=0}=\nabla_{\perp}^{2} \phi_{0}(\boldsymbol{x}) .
$$

To simplfy our notation, let us define

$$
b(x)=\frac{I(x, \Delta z)-I(x,-\Delta z)}{J_{0}}
$$

as our measurement, obtained by a centered finite difference around $z=0$ for $\partial I / \partial z$. We then specify TIE in the Fourier domain as

$$
\widehat{b}(\omega)=4 \pi \lambda \Delta z\|\omega\|_{2}^{2} \widehat{\phi_{0}}(\omega),
$$

where the Fourier transform of a function $f: \mathbb{R}^{2} \rightarrow \mathbb{C}$ is given by

$$
\begin{aligned}
\widehat{f}(\boldsymbol{\omega}) & =\mathcal{F}\{f\}(\boldsymbol{\omega}) \\
& =\int_{\mathbb{R}^{2}} f(\boldsymbol{x}) \mathrm{e}^{-\mathrm{j} 2 \pi \omega \bullet \boldsymbol{x}} \mathrm{d} \boldsymbol{x} .
\end{aligned}
$$

Consequently, the sought phase is obtained by

$$
\phi_{0}=\mathcal{F}^{-1}\left\{\widehat{b}_{\mathrm{TIE}}^{-1}\right\}
$$

where

$$
\widehat{h}_{\mathrm{TIE}}(\boldsymbol{\omega})= \begin{cases}\epsilon, & \boldsymbol{\omega}=\mathbf{0} \\ 4 \pi \lambda \Delta z\|\boldsymbol{\omega}\|_{2}^{2}, & \text { otherwise, }\end{cases}
$$

with $\epsilon>0$ being introduced to deal with the singularity at the origin. This makes the inverse filter $\widehat{h}_{\mathrm{TIE}}^{-1}$ well-defined. In practical terms, (10) explains that a conventional reconstruction of the phase requires one to capture three images (the infocus image recorded at $z=0$ and the two defocus images recorded at $z= \pm \Delta z$ ), and to use a Fourier-domain filtering operation.

We now would like to discuss how the defocus distance affects the reconstruction performance. From a mathematical point of view, $\Delta z$ should be as small as possible so that $\partial I / \partial z$ is well-approximated. However, in practice, the intuitive appeal of choosing a very small defocus is suboptimal. To see this, let us assume that the measurement $b$ contains some amount of white noise that does not depend on $\Delta z$. The spectrum of the noise in the reconstructed phase $\phi_{0}$ is 


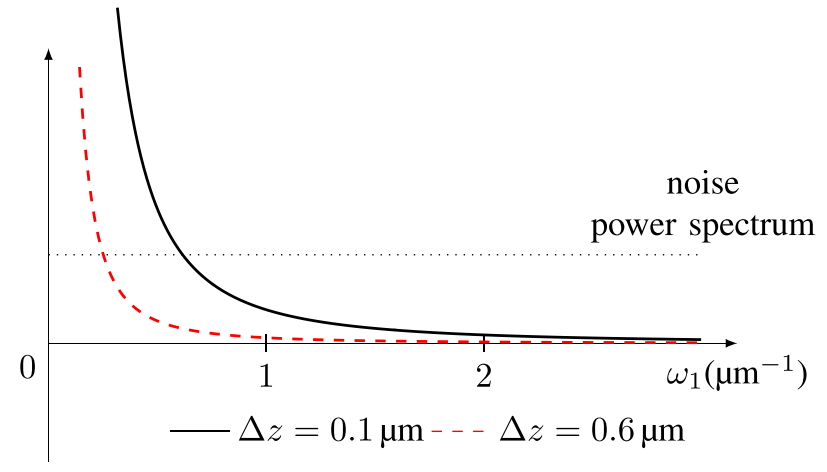

Fig. 3. Radial profile of the inverse transfer function $\widehat{h}_{\mathrm{TIE}}^{-1}$ for $\lambda=632 \mathrm{~nm}$ using two distinct defocus distances. For simplicity, the profile is shown only for positive spatial frequencies.

shaped by $\widehat{h}_{\mathrm{TIE}}^{-1}$ which is essentially a two fold integrator (since $\widehat{h}_{\mathrm{TIE}} \propto\|\boldsymbol{\omega}\|_{2}^{2}$ ). Therefore, the phase image (especially the lower spatial frequencies) is perturbed more as $\Delta z$ gets smaller (see Figure 3). This aspect of TIE is well-known and discussed in several works [13], [20], [28], [29].

In summary, by increasing the defocus distance, TIE-based methods resolve lower spatial frequencies better as noiseinduced artifacts are reduced. However, for large $\Delta z$, the implicit linearity in the finite difference approximation breaks and one obtains coarser $\partial I / \partial z$ estimates. It is thus reasonable to use large defocus measurements for reconstructing low frequencies as we rely on small defocus measurements for the high ones. To have a better understanding of how to combine the two regimes, we shall explore a complementary formulation.

\section{B. Contrast Transfer Function}

In the sequel, we base our approach on the principles of wave propagation. Under the paraxial approximation, the intensity of the wave field is expressed as

$$
\begin{aligned}
I(\boldsymbol{x}, z) & =|U(\boldsymbol{x}, z)|^{2} \\
& =|O(\boldsymbol{x}) * p(\boldsymbol{x}, z)|^{2},
\end{aligned}
$$

where $*$ denotes the convolution operator and

$$
p(\boldsymbol{x}, z)=\frac{\mathrm{e}^{\mathrm{j} k z}}{\mathrm{j} \lambda z} \exp \left(\frac{\mathrm{j} k}{2 z}\|\boldsymbol{x}\|_{2}^{2}\right)
$$

represents the impulse response of the Fresnel propagation [40]. We remark that

$$
\hat{p}(\omega, z)=\mathrm{e}^{\mathrm{j} k z} \mathrm{e}^{-\mathrm{j} \pi \lambda z\|\omega\|_{2}^{2}} .
$$

In effect, the term $\exp \left(-\mathrm{j} \pi \lambda z\|\omega\|_{2}^{2}\right)$ represents the optical transfer function for the propagation. The complex amplitude is given by $\widehat{U_{A}}(\omega, z)=\widehat{O}(\omega, z) \mathrm{e}^{-\mathrm{j} \pi \lambda z\|\omega\|_{2}^{2}}$.

As we are interested in imaging thin biological samples, we assume that $\phi_{0}$ is small. The object field is approximated by

$$
O(x) \approx \sqrt{J_{0}}\left(1+\mathrm{j} \phi_{0}(x)\right) .
$$

Then,

$$
\begin{aligned}
\widehat{U_{A}}(\omega, z) \approx \sqrt{J_{0}}(\delta(\omega)+ & \widehat{\phi_{0}}(\omega)\left(\mathrm{j} \cos \left(\pi \lambda z\|\omega\|_{2}^{2}\right)\right. \\
+ & \left.\left.\sin \left(\pi \lambda z\|\omega\|_{2}^{2}\right)\right)\right) .
\end{aligned}
$$

Also, we remark that

$$
\widehat{I}(\omega, z)=\left({\widehat{U_{A}}}^{*}(-\cdot, z) * \widehat{U_{A}}(\cdot, z)\right)(\boldsymbol{\omega})
$$

and that $\widehat{\phi}_{0}$ is Hermitian-symmetric since $\phi_{0}$ is real-valued. Then, by developing (17) up to the first-order term in $\phi_{0}$ (i.e., neglecting the higher-order terms that include the convolution $\left.\widehat{\phi_{0}} * \widehat{\phi_{0}}\right)$, we obtain

$$
\widehat{I}(\omega, z)=J_{0}\left(\delta(\omega)+2 \sin \left(\pi \lambda z\|\omega\|_{2}^{2}\right) \widehat{\phi}_{0}(\omega)\right),
$$

which is known as the contrast transfer function (CTF) [30]. As suggested by its name, the CTF explains how the phase and the propagation distance is changing the image contrast. For instance, in the present case, it points out that the visibility of phase-only objects is increased by introducing a suitable defocus (see Figure 7(a)). Evaluating (18) at points $z=\Delta z$ and $z=-\Delta z$ yields the TIE-like equation

$$
\widehat{b}(\omega)=4 \sin \left(\pi \lambda \Delta z\|\omega\|_{2}^{2}\right) \widehat{\phi}_{0}(\omega) .
$$

Similar to $\widehat{h}_{\text {TIE }}$ in (10), we define

$$
\widehat{h}_{\mathrm{CTF}}(\boldsymbol{\omega})= \begin{cases}\epsilon, & \boldsymbol{\omega}=\mathbf{0} \\ 4 \sin \left(\pi \lambda \Delta z\|\boldsymbol{\omega}\|_{2}^{2}\right), & \text { otherwise. }\end{cases}
$$

Considering $\widehat{h}_{\mathrm{TIE}}$ and $\widehat{h}_{\mathrm{CTF}}$, let us further develop our approach for the phase retrieval problem. First, it is important to notice that the models are undistinguishable when $\Delta z$ is sufficiently small. In contrast, as $\Delta z$ gets larger, the models still agree up to a certain frequency $\omega_{0}$, and differ afterwards (see Figure 4). The validity of utilizing TIE with large defocus measurements is enforced in the region where it agrees with the CTF model [31]. Above $\omega_{0}$, the two models tend to disagree and it is therefore safer to exclude these frequencies.

We shall use large defocus intensity images to recover low frequencies up to $\omega_{0}$ (denoted as "Region 1" in Figure 4). For the rest of the spectrum (denoted as "Region 2" in Figure 4), we rely on the small defocus images. We remark that similar approaches have been carried out in [20]-[23].

\section{ReCONSTRUCTION Algorithm}

In this section, we put our phase recovery problem in a variational framework. We shall work with two defocus distances and base our approach on a weighted reconstruction. To effectively combine low- and high- frequency components of the phase, we first construct the spectral weighting filters.

\section{A. Spectral Weighting Filters}

Considering the physical principles explained in Section II, we introduce two isotropic functions that are appropriate for the spectral weighting of the measurements. We denote these functions by $W_{\mathrm{LP}}$ and $W_{\mathrm{HP}}$, for low-pass and high-pass weighting, respectively. They are defined as follows:

$$
W_{\mathrm{LP}}(\boldsymbol{\omega})= \begin{cases}1, & \|\boldsymbol{\omega}\|_{2}<\omega_{0}-\frac{L}{2} \\ g(\boldsymbol{\omega}), & \omega_{0}-\frac{L}{2}<\|\boldsymbol{\omega}\|_{2}<\omega_{0}+\frac{L}{2} \\ 0, & \|\boldsymbol{\omega}\|_{2}>\omega_{0}+\frac{L}{2},\end{cases}
$$



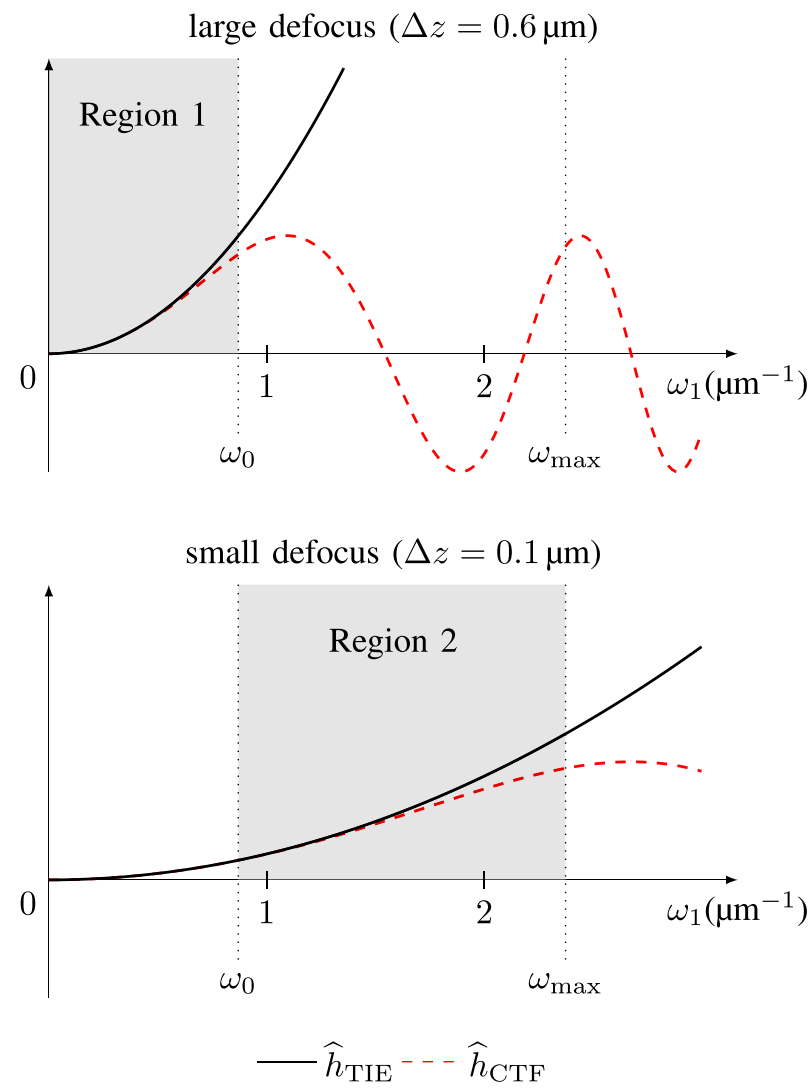

Fig. 4. Radial profile of the transfer functions for $\lambda=632 \mathrm{~nm}$. For simplicity, the profile is shown only for positive spatial frequencies.

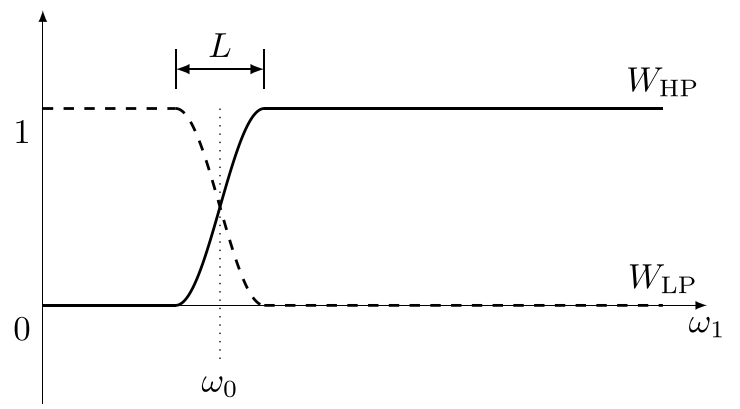

Fig. 5. Radial profile of the spectral filters used to weight low and high frequencies of the measurements, computed for two defocus distances.

where

$$
g(\omega)=\frac{1}{2}\left(1+\cos \left(\frac{\pi}{L}\left(\|\omega\|_{2}-\omega_{0}+\frac{L}{2}\right)\right)\right)
$$

and

$$
W_{\mathrm{HP}}(\omega)=1-W_{\mathrm{LP}}(\omega) .
$$

The parameter $\omega_{0}$ denotes the cutoff frequency; the values of $W_{\mathrm{HP}}$ and $W_{\mathrm{LP}}$ are equal for $\|\omega\|_{2}=\omega_{0}$. Note that (22) provides a smooth transition zone of width $L$ around $\omega_{0}$. A graphical representation of our filters is seen in Figure 5.

It is noteworthy that $\omega_{0}$ specifies the limit spatial frequency up to which the phase reconstruction based on a large defocus is reliable. For the frequencies that are higher than $\omega_{0}$, we shall rely on the measurements obtained with a small defocus. Considering (11) and (20), we define $\omega_{0}$ as

$$
\omega_{0}=\sqrt{\frac{\theta_{0}}{\pi \lambda \Delta z}}
$$

where $\theta_{0}$ is the critical value after which the difference between $\widehat{h}_{\mathrm{TIE}}$ and $\widehat{h}_{\mathrm{CTF}}$ is not negligible.

\section{B. Discrete Formulation}

We consider that two distinct defocus values, $\Delta z_{1}$ and $\Delta z_{2}$ with $\Delta z_{1}<\Delta z_{2}$, are used. Let $b_{1}$ and $b_{2}$ represent the corresponding measurements computed via (7) for $\Delta z_{1}$ and $\Delta z_{2}$, respectively. Since the measurements are only known at discrete pixel locations, we collect their sample values in the vectors $^{2} \mathbf{b}_{1}, \mathbf{b}_{2} \in \mathbb{R}^{N}$ with $N$ being the total number of pixels on the detector. Then, in the noise-free scenario, (8) implies that

$$
\begin{aligned}
& \mathbf{b}_{1}=\mathbf{H}_{1} \boldsymbol{\Phi}, \\
& \mathbf{b}_{2}=\mathbf{H}_{2} \boldsymbol{\Phi},
\end{aligned}
$$

where $\boldsymbol{\Phi} \in \mathbb{R}^{N}$ is the discretized version of the original phase map and $\mathbf{H}_{1}, \mathbf{H}_{2} \in \mathbb{R}^{N \times N}$ correspond to $h_{\mathrm{TIE}}$ for $\Delta z_{1}$ and $\Delta z_{2}$, respectively (thus, represent Laplacian operators up to different multiplicative factors). These operators are selfadjoint in the sense that $\mathbf{H}_{1}^{\mathrm{T}}=\mathbf{H}_{1}$ and $\mathbf{H}_{2}^{\mathrm{T}}=\mathbf{H}_{2}$, where $(\cdot)^{\mathrm{T}}$ denotes the matrix transpose.

We then define our weighting matrices as

$$
\begin{aligned}
& \mathbf{W}_{1}=\mathbf{F}^{\mathrm{H}} \boldsymbol{\Sigma}_{\mathrm{HP}} \mathbf{F}, \\
& \mathbf{W}_{2}=\mathbf{F}^{\mathrm{H}} \boldsymbol{\Sigma}_{\mathrm{LP}} \mathbf{F},
\end{aligned}
$$

where $\mathbf{F}$ is the discrete Fourier transform (DFT) matrix, $(\cdot)^{\mathrm{H}}$ denotes the Hermitian transpose, and $\boldsymbol{\Sigma}_{\mathrm{LP}}, \boldsymbol{\Sigma}_{\mathrm{HP}} \in \mathbb{R}^{N \times N}$ are diagonal matrices whose entries are the discrete samples of $W_{\mathrm{LP}}$ and $W_{\mathrm{HP}}$, respectively. In practice, a small positive constant can be added to the diagonal elements. This construction makes $\mathbf{W}_{1}$ and $\mathbf{W}_{2}$ positive-definite matrices.

We propose the data-fidelity term

$$
D(\boldsymbol{\Phi})=\frac{1}{2}\left\|\mathbf{H}_{1} \boldsymbol{\Phi}-\mathbf{b}_{1}\right\|_{\mathbf{W}_{1}}^{2}+\frac{1}{2}\left\|\mathbf{H}_{2} \boldsymbol{\Phi}-\mathbf{b}_{2}\right\|_{\mathbf{W}_{2}}^{2},
$$

where $\|\cdot\|_{\mathbf{W}_{r}}^{2}$ is the weighted norm defined as $\left\langle\mathbf{W}_{r} \cdot, \cdot\right\rangle$ for $r=1,2$. This corresponds to a maximum-likelihood (ML) functional under the hypothesis that the measurements are degraded by additive white Gaussian noise (AWGN) [32].

Based on this formalism, we aim obtaining a phase reconstruction $\tilde{\boldsymbol{\Phi}}$ such that

$$
\tilde{\boldsymbol{\Phi}}=\arg \min _{\boldsymbol{\Phi}}(D(\boldsymbol{\Phi})+\tau R(\boldsymbol{\Phi})),
$$

with $R$ being the regularization functional given by

$$
R(\boldsymbol{\Phi})=\sum_{i \in \mathcal{I}}\left\|\mathbf{L}_{i} \boldsymbol{\Phi}\right\|_{2}^{p},
$$

where $\mathcal{I}$ is the index set of all pixel locations, $p$ is a positive scalar value, and $\mathbf{L}$ is the discrete analogue of the transverse

\footnotetext{
${ }^{2}$ The vectorization of the measurements are assumed to be lexicographically ordered.
} 
gradient operator. Thus, $\mathbf{L}_{i} \Phi \in \mathbb{R}^{2}$ represents the firstorder finite differences of $\boldsymbol{\Phi}$ along the vertical and horizontal directions at location $i$. Our data term selectively incorporates the high- and low-frequency information coming from the measurements taken at $\pm \Delta z_{1}$ and $\pm \Delta z_{2}$, respectively. The regularizer imposes smoothness on the reconstructions, where the strength of the smoothness is controlled by the regularization parameter $\tau>0$.

Let us explain some special cases of the proposed framework. Discarding the spectral weighting (i.e., $\mathbf{W}_{1}$ and $\mathbf{W}_{2}$ are identity operators) and setting $p=2$, one obtains the classical Tikhonov regularization, which can be seen as the conventional way of solving TIE. Assuming periodic boundary conditions, these linear methods can be directly solved using the fast Fourier transform [33]. The case $p=1$ gives the total variation (TV) regularization [34], which has been considered in [25] and [27] in the context of TIE. More importantly, if weighting is nontrivial and Tikhonov regularization is considered, the solution is given by

$$
\tilde{\boldsymbol{\Phi}}_{\mathrm{Tik}}=\mathbf{A}^{-1}\left(\mathbf{H}_{1} \mathbf{W}_{1} \mathbf{b}_{1}+\mathbf{H}_{2} \mathbf{W}_{2} \mathbf{b}_{2}\right)
$$

where $\mathbf{A}=\left(\mathbf{H}_{1} \mathbf{W}_{1} \mathbf{H}_{1}+\mathbf{H}_{2} \mathbf{W}_{2} \mathbf{H}_{2}+2 \tau \mathbf{L}^{\mathrm{T}} \mathbf{L}\right)$. Note that $(30)$ can also be interpreted as some Wiener filter. As $\tau \rightarrow 0$ (i.e., no regularization), one recovers a method that is in spirit of [20]-[23]. We note that the matrix inversion in (30) is welldefined, even in the absence of regularization. However, in cell imaging applications, it is often desirable that the specimens be well-isolated from the background [12]. Therefore, we combine our data-fidelity term with a TV functional, which is known to preserve discontinuities better than Tikhonov-type regularizations [34].

For the phase-reconstruction problem, we propose the nonlinear optimization problem

$$
\begin{aligned}
\tilde{\boldsymbol{\Phi}}=\arg \min _{\boldsymbol{\Phi}}( & \frac{1}{2}\left\|\mathbf{H}_{1} \boldsymbol{\Phi}-\mathbf{b}_{1}\right\|_{\mathbf{W}_{1}}^{2} \\
& \left.+\frac{1}{2}\left\|\mathbf{H}_{2} \boldsymbol{\Phi}-\mathbf{b}_{2}\right\|_{\mathbf{W}_{2}}^{2}+\tau \sum_{i \in \mathcal{I}}\left\|\mathbf{L}_{i} \boldsymbol{\Phi}\right\|_{2}\right) .
\end{aligned}
$$

TV regularization can be suboptimal for phase profiles that do not exhibit discontinuities. In such cases, staircase effect can occur and thus a higher-order regularization should be incorporated into our model [35].

\section{Optimization Algorithm}

In the sequel, we are going to solve our specific phase reconstruction problem using generic optimization tools: the alternating-direction method of multipliers (ADMM) and proximal operators, which have recently become quite popular in the field [36]. We first cast (31) as a constrained optimization problem given by

$$
\begin{gathered}
\tilde{\boldsymbol{\Phi}}=\arg \min _{\boldsymbol{\Phi}}\left(\frac{1}{2}\left\|\mathbf{H}_{1} \boldsymbol{\Phi}-\mathbf{b}_{1}\right\|_{\mathbf{W}_{1}}^{2}+\frac{1}{2}\left\|\mathbf{H}_{2} \boldsymbol{\Phi}-\mathbf{b}_{2}\right\|_{\mathbf{W}_{2}}^{2}\right. \\
\left.+\tau \sum_{i \in \mathcal{I}}\left\|\mathbf{u}_{i}\right\|_{2}\right) \text { s.t. } \mathbf{u}=\mathbf{L} \boldsymbol{\Phi},
\end{gathered}
$$

where $\mathbf{u}$ is an auxiliary variable. To solve (32), we introduce the associated augmented Lagrangian functional

$$
\begin{aligned}
L_{\mathrm{A}}(\boldsymbol{\Phi}, \mathbf{u}, \boldsymbol{\alpha})= & \frac{1}{2}\left\|\mathbf{H}_{1} \boldsymbol{\Phi}-\mathbf{b}_{1}\right\|_{\mathbf{W}_{1}}^{2}+\frac{1}{2}\left\|\mathbf{H}_{2} \boldsymbol{\Phi}-\mathbf{b}_{2}\right\|_{\mathbf{W}_{2}}^{2} \\
& +\tau \sum_{i \in \mathcal{P}}\left\|\mathbf{u}_{i}\right\|_{2} \\
& -\boldsymbol{\alpha}^{\mathrm{T}}(\mathbf{u}-\mathbf{L} \boldsymbol{\Phi})+\frac{\beta}{2}\|\mathbf{u}-\mathbf{L} \boldsymbol{\Phi}\|_{2}^{2},
\end{aligned}
$$

where $\alpha$ is the Lagrange multiplier and $\beta>0$ is called the penalty parameter. We then use ADMM [36], [37], where the main idea is to individually treat $L_{\mathrm{A}}$ over each of its arguments while the others are assumed to be fixed. This results in an iterative framework whose steps are

$$
\begin{aligned}
& \mathbf{u}^{t+1}=\arg \min _{\mathbf{u}} L_{\mathrm{A}}\left(\boldsymbol{\Phi}^{t}, \mathbf{u}, \boldsymbol{\alpha}^{t}\right), \\
& \boldsymbol{\Phi}^{t+1}=\arg \min _{\boldsymbol{\Phi}} L_{\mathrm{A}}\left(\boldsymbol{\Phi}, \mathbf{u}^{t+1}, \boldsymbol{\alpha}^{t}\right), \\
& \boldsymbol{\alpha}^{t+1}=\boldsymbol{\alpha}^{t}-\beta\left(\mathbf{u}^{t+1}-\mathbf{L} \boldsymbol{\Phi}^{t+1}\right) .
\end{aligned}
$$

We note that the minimization over $\mathbf{u}$ in (34a) is separable and amounts to the proximal operator associated with $\|\cdot\|_{2}$ [38]. This implies that the solution is obtained by solving the $N$ minimization problems

$$
\mathbf{u}_{i}^{t+1}=\arg \min _{\mathbf{u}_{i}}\left(\tau\left\|\mathbf{u}_{i}\right\|_{2}+\frac{\beta}{2}\left\|\mathbf{u}_{i}-\mathbf{z}_{i}^{t}\right\|_{2}^{2}\right), \forall i \in \mathcal{I},
$$

with the closed-form solution being

$$
\mathbf{u}_{i}^{t+1}=\max \left\{\left\|\mathbf{z}_{i}^{t}\right\|_{2}-\frac{\tau}{\beta}, 0\right\} \frac{\mathbf{z}_{i}^{t}}{\left\|\mathbf{z}_{i}^{t}\right\|_{2}},
$$

where $\mathbf{z}_{i}^{t}=\mathbf{L}_{i} \boldsymbol{\Phi}^{t}+\frac{\boldsymbol{\alpha}_{i}^{t}}{\beta}$. Since the minimization can be implemented for each pixel location in parallel, $\mathbf{u}^{t+1}$ is computed efficiently.

The second subproblem (34b) has the form of a standard quadratic minimization. In this case, the solution is expressed as

$$
\boldsymbol{\Phi}^{t+1}=\left(\mathbf{H}_{1} \mathbf{W}_{1} \mathbf{H}_{1}+\mathbf{H}_{2} \mathbf{W}_{2} \mathbf{H}_{2}+\beta \mathbf{L}^{\mathrm{T}} \mathbf{L}\right)^{-1} \mathbf{M}^{t}
$$

where

$$
\mathbf{M}^{t}=\left(\mathbf{H}_{1} \mathbf{W}_{1} \mathbf{b}_{1}+\mathbf{H}_{2} \mathbf{W}_{2} \mathbf{b}_{2}+\beta \mathbf{L}^{\mathrm{T}}\left(\mathbf{u}^{t+1}-\frac{\boldsymbol{\alpha}^{t}}{\beta}\right)\right) .
$$

It is actually seen that a Tikhonov-type minimization is computed. Similar to (30), this problem is solved directly for certain boundary conditions (in other cases, iterative solutions are obtained via linear solvers). The last step (34c) is a standard update of the Lagrange multiplier.

\section{EXPERIMENTS}

We illustrate the utility of our phase reconstruction method by performing experiments in simulated and practical configurations. In every experiment, the images are extended using periodic boundary conditions. The $\epsilon$ parameter in (11) is set to 1 so that the filter $\widehat{h}_{\mathrm{TIE}}$ is mean-preserving. The $\theta_{0}$ parameter in (24) is set to $\pi / 10$, and the associated width $L$ is manually tuned according to the maximal frequency 

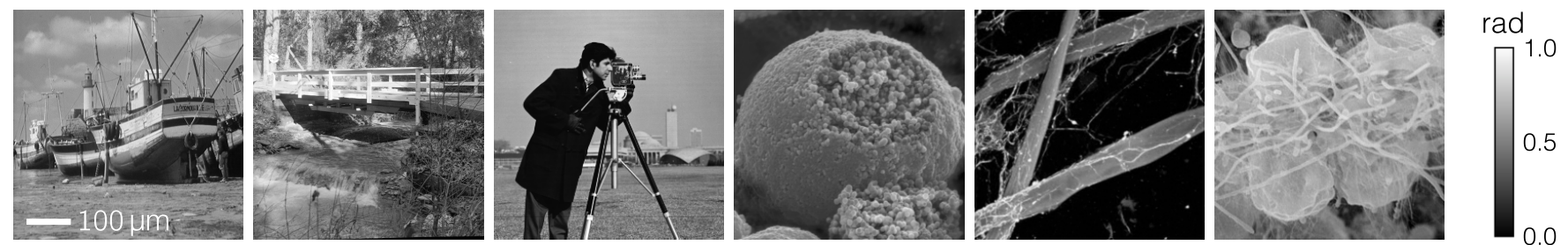

Fig. 6. Ground-truth spatial phase maps used in the simulations. From left to right, they are referred to as 1) Boat, 2) Bridge, 3) Cameraman, 4) CIL214, 5) CIL38921, and 6) CIL39789.

present in the signal. For synthetic data, this maximal frequency is set to the inverse of the pixel size; for real data experiments, it is the minimum of the inverse effective pixel size (i.e., the camera pixel size divided by the magnification) and the resolution imposed by the diffraction limit (i.e., twice the numerical aperture divided by the wavelength). For ADMM-based reconstructions, we fix $\beta=10 \tau$. We also fix the stopping criterion as reaching either a relative $\ell_{2}$-normed difference of $10^{-4}$ between two successive iterates, or a maximum of 250 iterations. All numerical schemes are implemented in MATLAB (The MathWorks, Inc., Natick, MA).

\section{A. Synthetic Data}

The original object field is assumed to have a unit intensity, and the spatial phase map is given by the set of two-dimensional images shown in Figure $6 .{ }^{3}$ All the groundtruth phase maps are of size $(256 \times 256)$ pixels and have values in the range of $[0,1]$ radians. We set the pixel size to $2 \mu \mathrm{m}$ and the wavelength $\lambda$ to $632 \mathrm{~nm}$. The original object field is propagated to axial distances of $\pm 50 \mu \mathrm{m}$ (small defocus) and $\pm 300 \mu \mathrm{m}$ (large defocus) by using a Fresnel diffraction kernel [39]. We generate the observed intensities by taking the square modulus of the propagated complex fields. Finally, the intensity images are degraded by various levels of AWGN. The standard deviation of the noise is chosen such that a given signal-to-noise ratio (SNR) is achieved. An example defocus stack is illustrated in Figure 7(a).

We measure the quality of the reconstructed phase maps by computing the regressed SNR - the regression is there to get rid of additive and multiplicative constants. Between the ground-truth map $\boldsymbol{\Phi}$ and a reconstructed one $\tilde{\boldsymbol{\Phi}}$, this measure is defined as

$$
\operatorname{SNR}(\boldsymbol{\Phi}, \tilde{\boldsymbol{\Phi}})=\max _{a, b \in \mathbb{R}} 10 \log \left(\frac{\|\boldsymbol{\Phi}\|_{2}^{2}}{\|\boldsymbol{\Phi}-(a \tilde{\boldsymbol{\Phi}}+b)\|_{2}^{2}}\right) .
$$

The performance of our nonlinear method is compared against the following algorithms:

1) Tikhonov regularization based TIE (TIE-Tik): We adapt a linear reconstruction scheme by setting $p=2$ in (29). The method uses three images, the infocus image and two symmetrically defocused images. We apply the method separately with each set of measurements acquired at $\pm 50 \mu \mathrm{m}$ and $\pm 300 \mu \mathrm{m}$ defocus.

\footnotetext{
${ }^{3}$ The biological images originate from the cell image database and are available at http://www.cellimagelibrary.org/
}
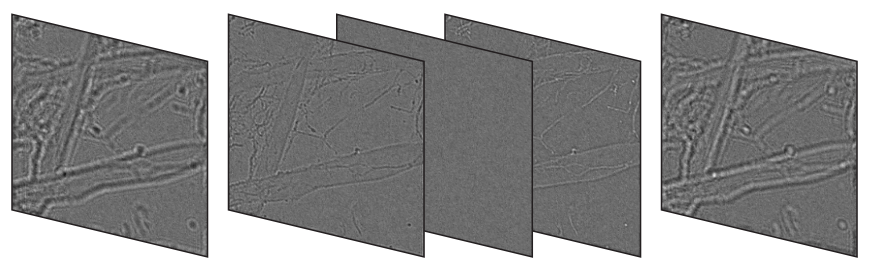

$-300 \mu \mathrm{m}$

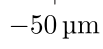

(a)
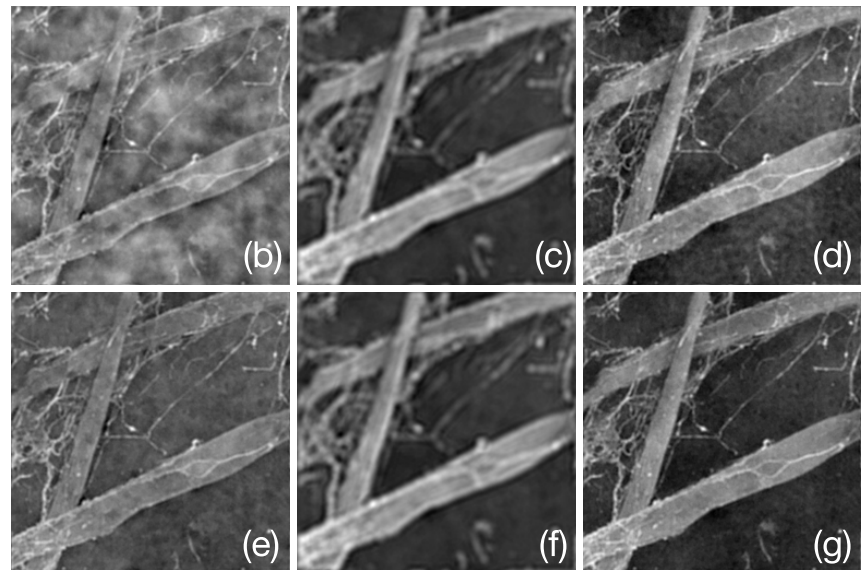

Fig. 7. Illustrative example of a phase-reconstruction simulation, where the object field (at $z=0$ ) has unit intensity and the phase is encoded by the CIL38921 image: (a) Simulated measurements (SNR is $20 \mathrm{~dB}$ ), (b) TIE-Tik reconstruction using the measurements at $50 \mu \mathrm{m}$ (SNR is $4.01 \mathrm{~dB}$ ), (c) TIE-Tik reconstruction using the measurements at $300 \mu \mathrm{m}$ (SNR is $8.67 \mathrm{~dB}$ ), (d) composite TIE-Tik reconstruction (SNR is $9.72 \mathrm{~dB}$ ), (e) TIE-TV reconstruction using the measurements at $50 \mu \mathrm{m}$ (SNR is $8.31 \mathrm{~dB}$ ), (f) TIE-TV reconstruction using the measurements at $50 \mu \mathrm{m}$ (SNR is $11.25 \mathrm{~dB}$ ), and $(\mathrm{g})$ proposed method (SNR is $13.20 \mathrm{~dB}$ ).

2) TV regularization based TIE (TIE-TV): We make the reconstruction nonlinear by setting $p=1$ in (29). Similarly, the algorithm uses three images and is tested with both small and large defocus cases.

3) Composite TIE-Tik: We adapt the reconstruction scheme given in (30). Composite TIE-Tik uses five images. It linearly combines high and low frequency components (together with a Tikhonov regularization) of the phase maps obtained by using small and large defocus measurements, respectively. The algorithm is compatible with the ones proposed in [20]-[23].

As described in Section III, all linear reconstructions are computed directly. The nonlinear ones are solved iteratively using the proposed ADMM-based algorithm. For each reconstruction algorithm, we tune the regularization parameter to 
TABLE I

Phase Reconstruction Performance of the Algorithms COMPARED in THE Simulations. Numbers ARE GIVEN IN DECIBEL UNIT

\begin{tabular}{|c|c|c|c|c|c|c|c|}
\hline & \multirow[b]{2}{*}{$\begin{array}{l}\text { Input } \\
\text { SNR }\end{array}$} & \multicolumn{2}{|c|}{$\Delta z=50 \mu \mathrm{m}$} & \multicolumn{2}{|c|}{$\Delta z=300 \mu \mathrm{m}$} & \multirow[b]{2}{*}{$\begin{array}{c}\text { Composite } \\
\text { TIE-Tik }\end{array}$} & \multirow[b]{2}{*}{$\begin{array}{l}\text { Proposed } \\
\text { method }\end{array}$} \\
\hline & & TIE-Tik & TIE-TV & TIE-Tik & TIE-TV & & \\
\hline ڤ్రి & $\begin{array}{l}20 \\
25 \\
30 \\
35 \\
40 \\
45 \\
50\end{array}$ & $\begin{array}{l}10.72 \\
11.64 \\
12.34 \\
14.16 \\
16.09 \\
18.08 \\
19.99\end{array}$ & $\begin{array}{l}12.26 \\
13.86 \\
15.24 \\
16.74 \\
17.98 \\
19.20 \\
20.19\end{array}$ & $\begin{array}{l}12.85 \\
14.52 \\
15.84 \\
16.78 \\
17.09 \\
17.19 \\
17.23\end{array}$ & $\begin{array}{l}14.52 \\
15.63 \\
16.32 \\
16.88 \\
17.11 \\
17.20 \\
17.23\end{array}$ & $\begin{array}{l}13.47 \\
15.94 \\
18.10 \\
19.88 \\
20.69 \\
20.95 \\
\mathbf{2 1 . 0 4}\end{array}$ & $\begin{array}{l}15.22 \\
17.19 \\
18.70 \\
20.01 \\
20.73 \\
20.96 \\
21.04\end{array}$ \\
\hline 苋 & $\begin{array}{l}20 \\
25 \\
30 \\
35 \\
40 \\
45 \\
50\end{array}$ & $\begin{array}{c}8.59 \\
9.51 \\
10.30 \\
12.40 \\
13.67 \\
15.68 \\
16.86\end{array}$ & $\begin{array}{l}10.56 \\
11.68 \\
12.40 \\
13.73 \\
14.74 \\
15.96 \\
16.89\end{array}$ & $\begin{array}{l}10.91 \\
12.39 \\
13.27 \\
13.99 \\
14.20 \\
14.28 \\
14.30\end{array}$ & $\begin{array}{l}11.99 \\
12.99 \\
13.44 \\
14.01 \\
14.22 \\
14.28 \\
14.31\end{array}$ & $\begin{array}{l}11.69 \\
13.95 \\
15.43 \\
16.77 \\
17.24 \\
17.39 \\
\mathbf{1 7 . 4 4}\end{array}$ & $\begin{array}{l}13.11 \\
14.88 \\
15.80 \\
16.84 \\
17.28 \\
17.40 \\
17.44\end{array}$ \\
\hline 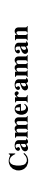 & $\begin{array}{l}20 \\
25 \\
30 \\
35 \\
40 \\
45 \\
50\end{array}$ & $\begin{array}{c}8.00 \\
9.77 \\
12.57 \\
14.77 \\
16.46 \\
17.52 \\
17.70\end{array}$ & $\begin{array}{l}13.83 \\
15.54 \\
16.38 \\
16.79 \\
17.23 \\
\mathbf{1 7 . 6 0} \\
\mathbf{1 7 . 7 2}\end{array}$ & $\begin{array}{l}13.17 \\
14.11 \\
14.43 \\
14.56 \\
14.61 \\
14.63 \\
14.63\end{array}$ & $\begin{array}{l}13.91 \\
14.26 \\
14.48 \\
14.55 \\
14.60 \\
14.62 \\
14.62\end{array}$ & $\begin{array}{l}14.37 \\
16.00 \\
16.82 \\
17.09 \\
17.18 \\
17.25 \\
17.24\end{array}$ & $\begin{array}{l}\mathbf{1 5 . 8 9} \\
\mathbf{1 6 . 6 7} \\
\mathbf{1 7 . 0 8} \\
\mathbf{1 7 . 1 8} \\
\mathbf{1 7 . 2 4} \\
17.29 \\
17.27\end{array}$ \\
\hline 竝 & $\begin{array}{l}20 \\
25 \\
30 \\
35 \\
40 \\
45 \\
50\end{array}$ & $\begin{array}{c}9.52 \\
10.60 \\
12.06 \\
13.34 \\
15.81 \\
18.11 \\
21.82\end{array}$ & $\begin{array}{l}11.87 \\
13.52 \\
14.86 \\
15.87 \\
17.81 \\
19.68 \\
21.97\end{array}$ & $\begin{array}{l}13.18 \\
14.59 \\
16.05 \\
17.22 \\
17.92 \\
18.08 \\
18.20\end{array}$ & $\begin{array}{l}14.82 \\
15.86 \\
16.73 \\
17.37 \\
17.96 \\
18.07 \\
18.19\end{array}$ & $\begin{array}{l}13.54 \\
15.72 \\
18.46 \\
21.16 \\
23.23 \\
24.05 \\
24.48\end{array}$ & $\begin{array}{l}15.12 \\
16.94 \\
19.22 \\
21.42 \\
23.35 \\
24.09 \\
24.49\end{array}$ \\
\hline 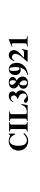 & $\begin{array}{l}20 \\
25 \\
30 \\
35 \\
40 \\
45 \\
50\end{array}$ & $\begin{array}{c}3.85 \\
5.30 \\
7.10 \\
9.75 \\
13.06 \\
15.34 \\
18.33\end{array}$ & $\begin{array}{c}8.30 \\
10.89 \\
13.41 \\
15.51 \\
17.54 \\
18.86 \\
20.51\end{array}$ & $\begin{array}{l}8.49 \\
10.64 \\
11.51 \\
12.46 \\
12.87 \\
12.96 \\
13.02\end{array}$ & $\begin{array}{l}11.15 \\
12.11 \\
12.48 \\
12.80 \\
12.95 \\
12.99 \\
13.02\end{array}$ & $\begin{array}{c}9.43 \\
13.13 \\
15.41 \\
18.52 \\
20.65 \\
21.35 \\
21.81\end{array}$ & $\begin{array}{l}12.63 \\
15.55 \\
17.88 \\
19.77 \\
21.11 \\
21.53 \\
21.84\end{array}$ \\
\hline 衣 & $\begin{array}{l}20 \\
25 \\
30 \\
35 \\
40 \\
45 \\
50\end{array}$ & $\begin{array}{l}11.92 \\
12.79 \\
14.22 \\
16.12 \\
18.48 \\
21.42 \\
25.93\end{array}$ & $\begin{array}{l}14.64 \\
17.22 \\
19.44 \\
21.47 \\
23.67 \\
25.55 \\
27.67\end{array}$ & $\begin{array}{l}16.29 \\
18.17 \\
19.81 \\
21.56 \\
22.35 \\
22.61 \\
22.73\end{array}$ & $\begin{array}{l}18.89 \\
20.46 \\
21.34 \\
22.14 \\
22.48 \\
22.65 \\
22.75\end{array}$ & $\begin{array}{l}16.57 \\
19.18 \\
21.77 \\
24.96 \\
27.09 \\
28.25 \\
28.65\end{array}$ & $\begin{array}{l}19.24 \\
22.15 \\
24.58 \\
26.22 \\
27.38 \\
28.40 \\
28.68\end{array}$ \\
\hline
\end{tabular}

achieve the best-possible SNR performance using an oracle. The output SNRs given in Table I are averaged over 10 realizations for a reliable comparison.

For the simulated measurements, we see that the proposed method outperforms the other algorithms in almost all of the cases. Especially for moderate and high levels of noise, our method significantly improves the reconstruction quality. A visual inspection of the reconstructed phase maps (see Figure 7) demonstrates that our method is able to reconstruct the high-frequency components accurately. However, the reconstructions using intensity images taken at large defocus distances are notably blurred. We also see that our algorithm produces much fewer artifacts in the low-frequency regions. Reconstruction errors are more visible for the other methods. As one gets to very low levels of noise, TIE-TV (for small defocus) and composite TIE-Tik methods become competitive, and TIE-TV reconstruction can yield the best results for certain configurations. This is explained by the decreased presence of noise-induced errors (i.e., low-frequency artifacts). Next, we assess our method in experimental settings to corroborate our in silico findings.

\section{B. Real Data}

We imaged paraformaldehyde-fixed and unstained $\mathrm{HeLa}$ cells at room temperature $\left(\sim 22^{\circ} \mathrm{C}\right)$. Acquisitions were performed on a Zeiss Axio Observer Z1 microscope (Carl Zeiss AG, Jena, Germany) equipped with a Leica HCX PL Fluotar $40 \times 0.75$ NA objective (Leica Microsystems $\mathrm{GmbH}$, Wetzlar, Germany). The camera pixel size is $6.5 \mu \mathrm{m}$ and the illumination wavelength is $684.5 \mathrm{~nm}$. The defocus images were recorded with distances of $\pm 2 \mu \mathrm{m}$ and $\pm 10 \mu \mathrm{m}$ from the best focal position.

A region of interest $(\mathrm{ROI})$ of size $(512 \times 512)$ pixels is chosen. Based on the previous results, we compare the reconstruction performance of TIE-Tik, TIE-TV, composite TIE-Tik, and the proposed method in a qualitative manner. We note that both TIE-Tik and TIE-TV methods use the measurements recorded at $\pm 2 \mu \mathrm{m}$. Since the ground-truth phase is not available, the regularization parameters are manually tuned (by paying attention to image contrast and physiological relevance) for all considered methods. The algorithmic settings of Section IV-A are kept the same for the reconstruction.

All TIE reconstructions improve the visibility of the cell compared to the infocus bright-field image. However, a closer examination reveals that the proposed approach enhances the homogeneity of the background better than its competitors. It allows one to better distinguish the cell membrane (see Figure 8). These aspects suggest that the proposed TIE-based approach is suitable for imaging phase-only objects.

\section{Validation}

Our final step is to compare different phase imaging techniques. To have a reference, the HeLa cells are imaged with a digital holographic microscope (DHM) that allows to directly obtain the complex object field. The hologram image is acquired in off-axis configuration on a T-1001 microscope (Lyncée Tec SA, Lausanne, Switzerland) using a Leica HI PLAN I $10 \times 0.22$ NA objective (Leica Microsystems GmbH, Wetzlar, Germany). The DHM image is then reconstructed numerically using the algorithm in [10]. A stack of brightfield images is acquired using the same objective. Following the lead of Kou et al. [15], we choose to apply our TIE formalism to differential interference contrast (DIC) images. These authors have investigated the DIC image formation model (under the thin phase-only object assumption) and have demonstrated the applicability of (11) for this modality (see [15] for further details). Practically observing the implications of (20), we thus acquire DIC images using an A-Plan $10 \times 0.25$ NA with a DIC analyser II. Both image stacks include the infocus image as well as images recorded at $\pm 2 \mu \mathrm{m}$ and $\pm 10 \mu \mathrm{m}$ defocus distances. Since the DHM measurements are performed on a different setup, we have been unable to align the exact same ROI. Instead, we choose a common ROI of size $(256 \times 256)$ in all images. We perform TIE reconstructions using our method on the chosen ROI.

We remark that the bright-field image has very low contrast, as explained in Section I. Meanwhile, DIC microscopes increase the contrast (also the visibility) of the cells; the final image can be roughly seen as the directional derivative of 


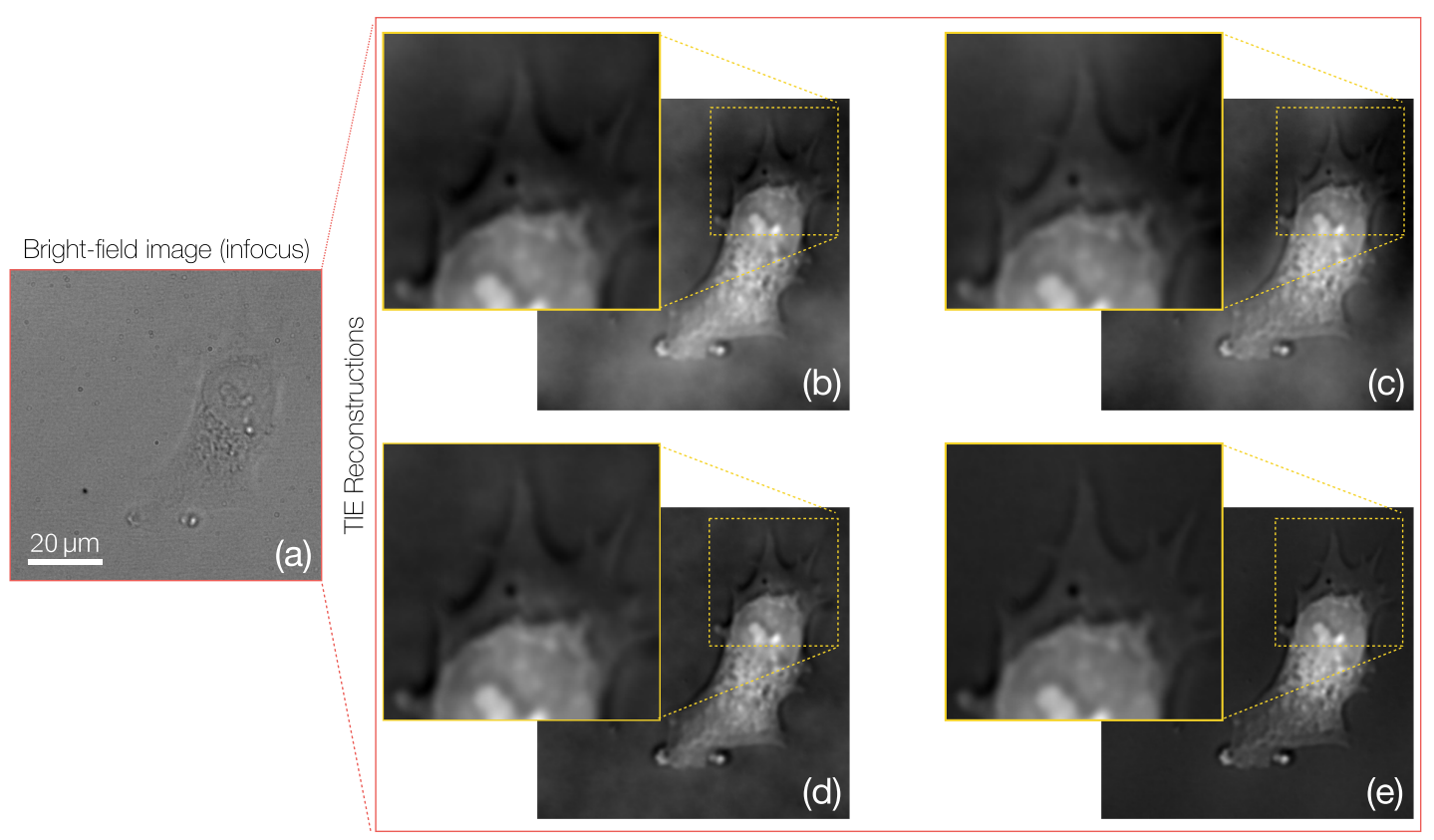

Fig. 8. TIE reconstruction results of different algorithms for experimentally acquired bright-field images of a HeLa cell: (a) Infocus bright-field image, (b) TIE-Tik reconstruction with defocus images acquired at $\pm 2 \mu \mathrm{m}$, (c) composite TIE-Tik reconstruction, (d) TIE-TV reconstruction with defocus images acquired at $\pm 2 \mu \mathrm{m}$, and (e) the proposed phase reconstruction method.
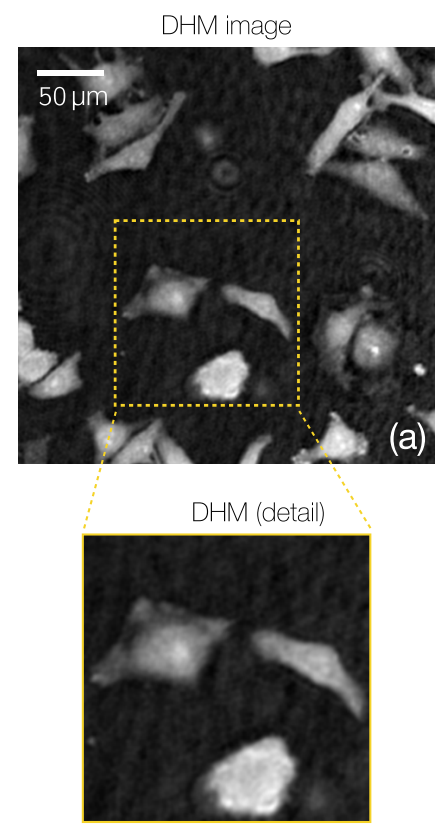

Bright-field image (infocus)
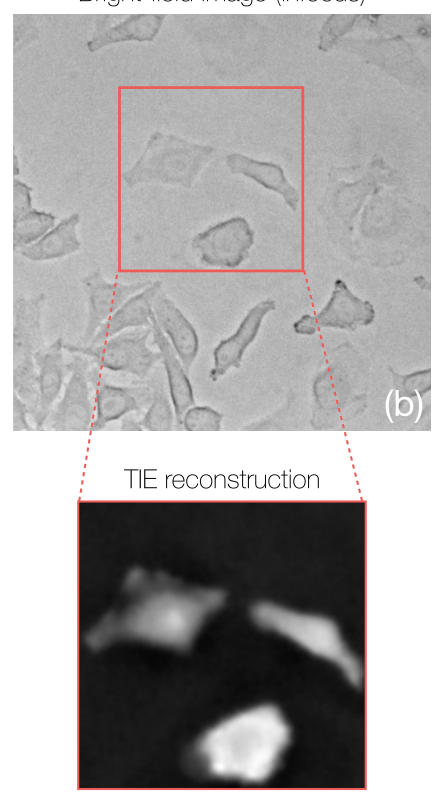

DIC image (infocus)
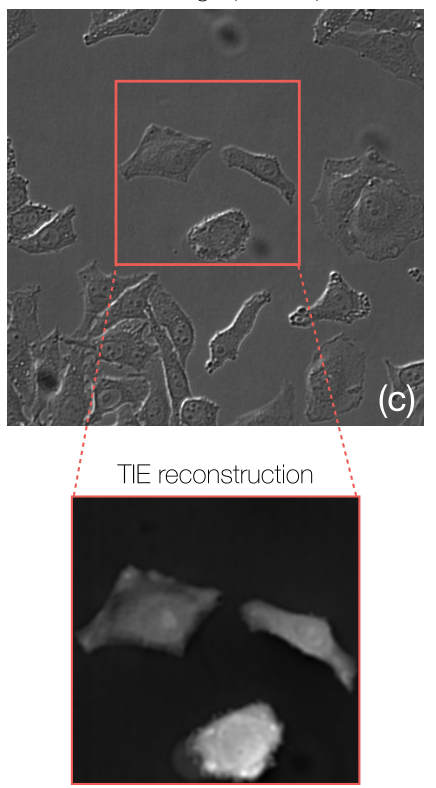

Fig. 9. Validation of the proposed TIE reconstruction method on the same sample: (a) Reference digital holographic microscope (DHM) image of HeLa cells, (b) result of the TIE reconstruction using bright-field images, and (c) result of the TIE reconstruction using differential interference contrast (DIC) images.

the phase. Note that DHM records a hologram of the object (i.e., single image acquisition) and produces the phase image by demodulating the hologram, which is a linear operation. However, DHM requires dedicated hardware and is much costlier compared than bright-field and DIC microscopes. By qualitatively looking at the results given in Figure 9, we see that the two TIE reconstructions render the morphology of the cells faithfully in accordance with the DHM image. We remark that TIE with DIC images produces sharper results than its counterpart using bright-field images. It is also seen that the TIE reconstructions have homogeneous backgrounds.
Hence, TIE can be considered as a cost-effective alternative to DHM.

As last illustration of the potential of our technique, we consider the problem of cell segmentation (i.e., automatic delineation of the cell boundaries). We choose a larger ROI and use the bright-field images for phase reconstruction. We apply the watershed segmentation algorithm. ${ }^{4}$ The segmentation results given in Figure 10 are seen to be satisfactory. By applying this basic segmentation algorithm to bright-field

\footnotetext{
${ }^{4}$ Available at http://bigwww.epfl.ch/sage/soft/watershed/
} 


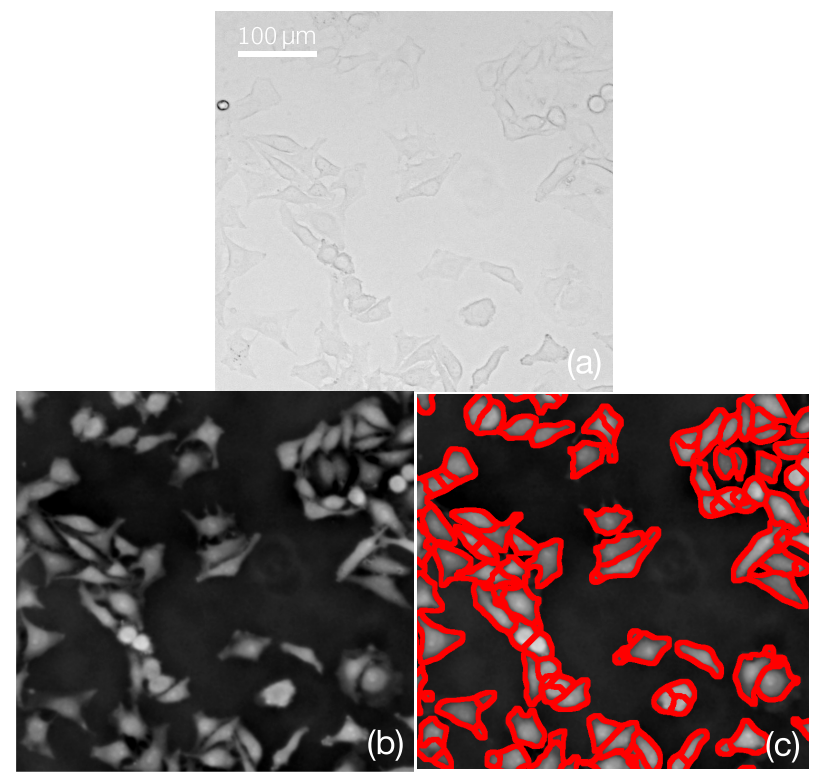

Fig. 10. Delineation of HeLa cells: (a) Infocus bright-field image, (b) phase image obtained by using the proposed TIE reconstruction, and (c) result of watershed algorithm.

images, it is not possible to achieve the same results as the bright-field images lack contrast.

\section{CONClusion}

We have introduced a new nonlinear algorithm for the optical phase retrieval problem. We have based our approach on the transport-of-intensity equation (TIE). We have investigated the implications of using TIE with measurements at small and large defocus distances in terms of the spatial frequencies of the reconstructed phase map. This has led to the formulation of a weighted phase-reconstruction algorithm. By acquiring measurements at two defocus distances and using our method, we have been able to improve the performance of phase reconstruction, as compared to previous algorithms. We have also shown that our method is applicable to experimental data. Finally, we have illustrated that the proposed method is beneficial to image analysis.

\section{ACKNOWLEDGMENTS}

The authors would like to thank Benjamin Rappaz from the Biomolecular Screening Facility, EPFL, and Etienne Shaffer from the Microsystems Laboratory 1, EPFL, for their help in acquiring the experimental data.

\section{REFERENCES}

[1] D. A. Boas, C. Pitris, and N. Ramanujam, Handbook of Biomedical Optics. Boca Raton, FL, USA: CRC Press, 2011.

[2] E. Betzig et al., "Imaging intracellular fluorescent proteins at nanometer resolution," Science, vol. 313, no. 5793, pp. 1642-1645, 2006.

[3] M. J. Rust, M. Bates, and X. Zhuang, "Sub-diffraction-limit imaging by stochastic optical reconstruction microscopy (STORM)," Nature Methods, vol. 3, no. 10, pp. 793-796, 2006.

[4] D. J. Stephens and V. J. Allan, "Light microscopy techniques for live cell imaging," Science, vol. 300, no. 5616, pp. 82-86, 2003

[5] G. Popescu, Quantitative Phase Imaging of Cells and Tissues. New York, NY, USA: McGraw-Hill, 2011.
[6] J. Marrison, L. Räty, P. Marriott, and P. O'Toole, "PtychographyA label free, high-contrast imaging technique for live cells using quantitative phase information," Sci. Rep., vol. 3, Aug. 2013, Art. ID 2369.

[7] F. Zernike, "Phase contrast, a new method for the microscopic observation of transparent objects," Physica, vol. 9, no. 7, pp. 686-698, 1942.

[8] G. Nomarski, "Nouveau dispositif pour l'observation en contraste de phase différentiel," J. Phys. Radium, vol. 16, no. 5, p. S88, 1955.

[9] E. Meijering, "Cell segmentation: 50 years down the road [life sciences]," IEEE Signal Process. Mag., vol. 29, no. 5, pp. 140-145, Sep. 2012.

[10] P. Marquet et al., "Digital holographic microscopy: A noninvasive contrast imaging technique allowing quantitative visualization of living cells with subwavelength axial accuracy," Opt. Lett., vol. 30, no. 5, pp. 468-470, 2005.

[11] M. M. Marim, M. Atlan, E. Angelini, and J.-C. Olivo-Marin, "Compressed sensing with off-axis frequency-shifting holography," Opt. Lett., vol. 35, no. 6, pp. 871-873, 2010.

[12] B. Rappaz, B. Breton, E. Shaffer, and G. Turcatti, "Digital holographic microscopy: A quantitative label-free microscopy technique for phenotypic screening," Combinat. Chem. High Throughput Screening, vol. 14, no. 1, pp. 80-88, 2014.

[13] M. R. Teague, "Deterministic phase retrieval: A Green's function solution," J. Opt. Soc. Amer., vol. 73, no. 11, pp. 1434-1441, 1983.

[14] D. Paganin and K. A. Nugent, "Noninterferometric phase imaging with partially coherent light," Phys. Rev. Lett., vol. 80, pp. 2586-2589, Mar. 1998.

[15] S. S. Kou, L. Waller, G. Barbastathis, and C. J. R. Sheppard, "Transportof-intensity approach to differential interference contrast (TI-DIC) microscopy for quantitative phase imaging," Opt. Lett., vol. 35, no. 3, pp. $447-449,2010$.

[16] A. Barty, K. A. Nugent, D. Paganin, and A. Roberts, "Quantitative optical phase microscopy," Opt. Lett., vol. 23, no. 11, pp. 817-819, 1998.

[17] L. Waller, L. Tian, and G. Barbastathis, "Transport of intensity phaseamplitude imaging with higher order intensity derivatives," Opt. Exp., vol. 18, no. 12, pp. 12552-12561, 2010.

[18] A. Pan, J. W. Lee, L. Waller, and G. Barbastathis, "Transport of intensity imaging with wavelet intensity derivative estimation," in Proc. Digit. Hologr. Three-Dimensional Imag., 2013, p. DW2A.20.

[19] Z. Jingshan, R. A. Claus, J. Dauwels, L. Tian, and L. Waller, "Transport of intensity phase imaging by intensity spectrum fitting of exponentially spaced defocus planes," Opt. Exp., vol. 22, no. 9, pp. 10661-10674, 2014.

[20] D. Paganin, A. Barty, P. J. McMahon, and K. A. Nugent, "Quantitative phase-amplitude microscopy. III. The effects of noise," J. Microscopy, vol. 214, no. 1, pp. 51-61, 2004.

[21] C.-Y. Chou, Y. Huang, D. Shi, and M. A. Anastasio, "Image reconstruction in quantitative X-ray phase-contrast imaging employing multiple measurements," Opt. Exp., vol. 15, no. 16, pp. 10002-10025, 2007.

[22] C. Zuo, Q. Chen, Y. Yu, and A. Asundi, "Transport-of-intensity phase imaging using Savitzky-Golay differentiation filter-Theory and applications," Opt. Exp., vol. 21, no. 5, pp. 5346-5362, 2013.

[23] J. Martinez-Carranza, K. Falaggis, and T. Kozacki, "Multi-filter transport of intensity equation solver with equalized noise sensitivity," Opt. Exp., vol. 23, no. 18, pp. 23092-23107, 2015.

[24] M. Mitome, K. Ishizuka, and Y. Bando, "Quantitativeness of phase measurement by transport of intensity equation," J. Electron Microscopy, vol. 59, no. 1, pp. 33-41, 2010.

[25] L. Tian, J. C. Petruccelli, and G. Barbastathis, "Nonlinear diffusion regularization for transport of intensity phase imaging," Opt. Lett., vol. 37, no. 19, pp. 4131-4133, 2012.

[26] E. Froustey, E. Bostan, S. Lefkimmiatis, and M. Unser, "Digital phase reconstruction via iterative solutions of transport-of-intensity equation," in Proc. IEEE 13th Workshop Inf. Opt. (WIO), Neuchâtel, Switzerland, Jul. 2014, pp. 1-3.

[27] E. Bostan, E. Froustey, B. Rappaz, E. Shaffer, D. Sage, and M. Unser, "Phase retrieval by using transport-of-intensity equation and differential interference contrast microscopy," in Proc. IEEE Int. Conf. Image Process. (ICIP), Paris, France, Oct. 2014, pp. 3939-3943.

[28] M. Soto, E. Acosta, and S. Ríos, "Performance analysis of curvature sensors: Optimum positioning of the measurement planes," Opt. Exp., vol. 11, no. 20, pp. 2577-2588, 2003.

[29] J. Martinez-Carranza, K. Falaggis, and T. Kozacki, "Optimum plane selection for transport-of-intensity-equation-based solvers," Appl. Opt., vol. 53, no. 30, pp. 7050-7058, 2014. 
[30] J. Frank, Three-Dimensional Electron Microscopy of Macromolecular Assemblies. New York, NY, USA: Academic, 1996.

[31] J. P. Guigay, M. Langer, R. Boistel, and P. Cloetens, "Mixed transfer function and transport of intensity approach for phase retrieval in the Fresnel region," Opt. Lett., vol. 32, no. 12, pp. 1617-1619, 2007.

[32] F. Aguet, D. Van De Ville, and M. Unser, "Model-based 2.5-D deconvolution for extended depth of field in brightfield microscopy," IEEE Trans. Image Process., vol. 17, no. 7, pp. 1144-1153, Jul. 2008.

[33] P. C. Hansen, J. G. Nagy, and D. P. O'Leary, Deblurring Images: Matrices, Spectra, and Filtering. Philadelphia, PA, USA: SIAM, 2006.

[34] L. I. Rudin, S. Osher, and E. Fatemi, "Nonlinear total variation based noise removal algorithms," Phys. D, Nonlinear Phenomena, vol. 60, nos. 1-4, pp. 259-268, 1992.

[35] S. Lefkimmiatis, A. Bourquard, and M. Unser, "Hessian-based norm regularization for image restoration with biomedical applications," IEEE Trans. Image Process., vol. 21, no. 3, pp. 983-995, Mar. 2012.

[36] S. Boyd, N. Parikh, E. Chu, B. Peleato, and J. Eckstein, "Distributed optimization and statistical learning via the alternating direction method of multipliers," Found. Trends Mach. Learn., vol. 3, no. 1, pp. 1-122, Jan. 2011.

[37] M. V. Afonso, J. M. Bioucas-Dias, and M. A. T. Figueiredo, "An augmented Lagrangian approach to the constrained optimization formulation of imaging inverse problems," IEEE Trans. Image Process., vol. 20 , no. 3, pp. 681-695, Mar. 2011

[38] P. L. Combettes and J.-C. Pesquet, "Proximal splitting methods in signal processing," in Fixed-Point Algorithms for Inverse Problems in Science and Engineering. New York, NY, USA: Springer-Verlag, 2011, pp. 185-212.

[39] M. Liebling, T. Blu, and M. Unser, "Fresnelets: New multiresolution wavelet bases for digital holography," IEEE Trans. Image Process., vol. 12, no. 1, pp. 29-43, Jan. 2003.

[40] J. W. Goodman, Introduction to Fourier Optics. New York, NY, USA: McGraw-Hill, 1996.

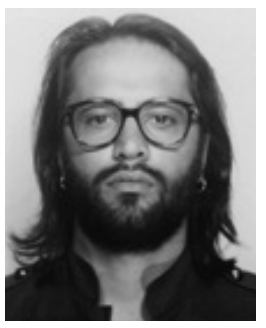

Emrah Bostan (S'11) received the B.Sc. degree in telecommunication engineering from Istanbul Technical University, Turkey, in 2009, and the M.Sc. degree in electrical engineering from the École Polytechnique Fédérale de Lausanne (EPFL), Lausanne, Switzerland, in 2011. He is currently pursuing the $\mathrm{Ph} . \mathrm{D}$. degree with the Biomedical Imaging Group, EPFL. His current research interests include inverse problems, variational models, and computational imaging.

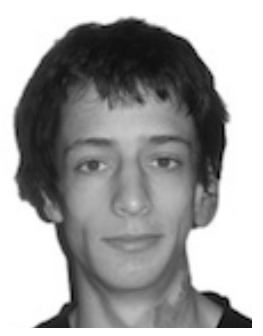

Emmanuel Froustey received the Engineering Diploma degree from CentraleSupélec, Châtenay-Malabry, France, in 2012, and the M.Sc. degree in computational science and engineering from the École Polytechnique Fédérale de Lausanne, Lausanne, Switzerland, in 2014. He is currently a Research and Development Engineer with Dassault Aviation. His research interests include variational models and optimization algorithms with applications to biomedical imaging.

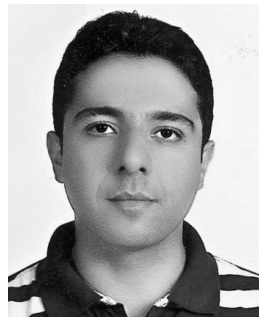

Masih Nilchian (S'10) received the M.S. degree in electrical engineering and communication system from the Sharif University of Technology, Tehran, Iran, in 2008, and the Ph.D. degree from the Biomedical Imaging Group, Swiss Federal Institute of Technology, Lausanne, Switzerland, under the direction of Prof. M. Unser, in 2015. He was involved in the applications of information and coding theory in biology and bioinformatics project in information system and security laboratory, Sharif University. From 2008 to 2010, he was a Research Officer with the MIMOS Laboratory, University Technology Malaysia. $\mathrm{He}$ is currently a Post-Doctoral Researcher with the Biomedical Imaging Group. His main research area is in phase retrieving in differential phase contrast and phase contrast X-ray computed tomography. He has interest in the use of spline for image processing, information theory, coding theory, wavelet and multiresolution algorithms, and iterative methods for reconstruction.

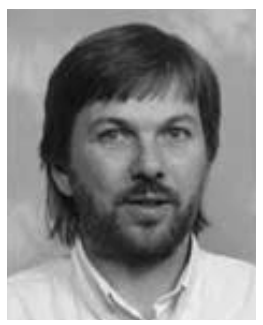

Daniel Sage received the M.S. and Ph.D. degrees in control and signal processing from the Institut National Polytechnique de Grenoble, Grenoble, France, in 1986 and 1989, respectively. From 1989 to 1998 , he was a Consulting Engineer. During his career, he has developed vision systems oriented to the quality control in the industrial sector. In 1998, he joined the Biomedical Imaging Group, École Polytechnique Fédérale de Lausanne, Lausanne, Switzerland, as the Head of Software Development. He is in charge of both the coordination of software development and of setting down the computing infrastructure of the group. He is also involved in the development of open-source bioimaging software (super-resolution, tracking, deconvolution, segmentation, and quantification), and methods for computer-assisted teaching.

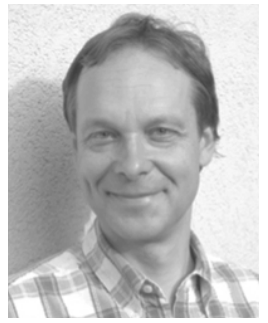

Michael Unser (M'89-SM'94-F'99) received the M.S. (summa cum laude) and Ph.D. degrees in electrical engineering from the École Polytechnique Fédérale de Lausanne (EPFL), Switzerland, in 1981 and 1984 , respectively. From 1985 to 1997 , he was a Scientist with the National Institutes of Health, Bethesda USA. He is currently a Full Professor and the Director of the Biomedical Imaging Group at EPFL.

He has published 200 journal papers on those topics and is one of the ISIs Highly Cited authors in engineering. His main research area is biomedical image processing. He has a strong interest in sampling theories, multiresolution algorithms, wavelets, and the use of splines for image processing.

Dr. Unser has held the position of Associate Editor-in-Chief (2003-2005) of the IEEE TRANSACTIONS ON MEDICAL IMAGING and has served as an Associate Editor for the IEEE TRANSACTIONS ON MEDICAL IMAGING (1999- 2002; 2006-2007), the IEEE TRANS ACTIONS ON IMAGE PROCESSING (1992-1995), and the IEEE Signal Processing Letters (1994-1998). $\mathrm{He}$ is currently a member of the Editorial Boards of Foundations and Trends in Signal Processing, and Sampling Theory in Signal and Image Processing. $\mathrm{He}$ co-organized the first IEEE International Symposium on Biomedical Imaging (ISBI2002) and was the Founding Chair of the Technical Committee of the IEEE-SP Society on Bio Imaging and Signal Processing.

Dr. Unser received the 1995 and 2003 Best Paper Awards, the 2000 Magazine Award, and two IEEE Technical Achievement Awards (2008 SPS and 2010 EMBS). He is a EURASIP Fellow and a member of the Swiss Academy of Engineering Sciences. 